\title{
Environment and infant immunity
}

\author{
Bengt Björkstén \\ Department of Health and Environment, Division of Paediatrics, University Hospital, Linköping University, \\ S-581 85 Linköping, Sweden
}

\begin{abstract}
Pregnancy is associated with a skewing towards T-helper (Th)2-like cell populations. During the first years of life, the neonatal immune responses towards allergens deviate towards a balanced Th1 and Th2-like immunity. The difference between atopic and nonatopic individuals may be how readily the immune deviation takes place. The high prevalence of allergies in industrialized countries is in contrast with the low prevalence of allergies in Eastern Europe, with a life style similar to that prevailing in Western Europe 40 years ago. The discussion on the impact of environmental changes on the incidence of asthma and other allergies has been limited mostly to the possible effects of a deteriorating air quality, poorly ventilated houses and an increased exposure to certain allergens, notably house dust mites. None of these factors can more than marginally explain the observed regional differences in the prevalence of allergic diseases. The concept of 'life style' should therefore be expanded considerably. The mother is a significant 'environmental factor' in early infancy. Human milk contains components that enhance the maturation of the immune system of the newborn infant. However, there are considerable individual variations in the composition of human milk. Recent studies indicate an imbalance in the gut flora of allergic, compared with non allergic infants, and in Swedish children compared with Estonian children. As the microbial flora drives the maturation of the immune system, changes in its composition may play a role for the higher prevalence of allergy. The future search for significant environmental factors should be directed towards other areas that have not yet been explored. The intestinal microflora is one of these factors that deserve a closer analysis.
\end{abstract}

\section{Allergy: Environment: Microflora: Human milk}

The relative risk for allergic disease and the tendency to develop allergic disease are both strongly influenced by genetic factors, age and the conditions under which exposure takes place. The likelihood of developing allergic disease in an individual is not constant over time, even if the general propensity is genetically determined. Thus, the risk of sensitization is affected by the period of exposure to allergens and the conditions under which exposure takes place. There seems to be a period in early life during which an infant is particularly susceptible to sensitization (Björkstén, 1997). For example, a higher incidence of allergy to birch (Betula spp.) and grass pollen has been reported in children born before the main pollen season (Björkstén \& Suoniemi, 1981). Similarly, neonatal exposure to cow's milk proteins is associated with elevated levels of immunoglobulin (Ig) $\mathrm{G}$ antibodies, at least during the first 8 years of life (Jenmalm \& Björkstén, 1999). The relationship between season of birth and development of allergy seems to be limited to children with a congenital propensity to develop allergic disease (Croner \& Kjellman, 1986). Similarly, the relationship between early feeding with foreign proteins and food allergy in infancy is seen particularly in genetically-susceptible persons (Björkstén, 1997).

\section{Development of immune responses to allergens}

Immune responses to allergens are characterized by a crossregulation between competing interferon $\gamma$-secreting T-helper (Th) 1-like cell populations and interleukin 4-producing Th2-like cell populations. Pregnancy is associated with a strong skewing towards Th2-type immunity, and if this change does not occur in time, there is an increased risk for abortion (Wegmann et al. 1993). As a consequence, neonatal immunity is Th2-skewed, and allergen specific $\mathrm{T}$-cell responses are already common at birth (Holt et al. 1997). Probably, these responses are normal and do not indicate future allergy. During the first years of life, the neonatal immune responses towards inhalant allergens deviates towards a balanced Th1 and Th2-like immunity, resulting in low levels of IgG antibodies and low-level T-cell responses to inhalant allergens in nonallergic children and adults. These T-cell responses are 
predominantly Th1-like. In contrast, immune responses to foods are generally suppressed. In a prospective study, the development of serum IgE and IgG subclass antibodies to $\beta$-lactoglobulin, ovalbumin, birch pollen and cat was analysed from birth up to 8 years of age (Jenmalm \& Björkstén, 1999). The levels of IgG subclass antibodies to $\beta$-lactoglobulin peaked at 6 months and to ovalbumin at 18 months and then decreased, while the IgG subclass antibody levels to the inhalant allergens generally increased with age. The kinetics of IgG antibodies to allergens were largely similar in Sweden which has a high prevalence of allergic disease, and in Estonia which has a low prevalence of allergic disease, except the Estonian babies had low levels of maternally-derived $\mathrm{IgG}_{4}$ antibodies to the inhalant allergens in cord blood (Julge, 1998). This finding indicates that these antibody responses are normally down-regulated. Atopic symptoms among Swedish children were associated with high levels of $\mathrm{IgG}$ antibodies, especially $\operatorname{IgG}_{4}$, to the allergens. The largest differences between atopic and nonatopic children were recorded at 6 months of age for the food allergens. For the seasonal allergen, birch pollen, the differences increased from 18 months of age. Thus, an early $\mathrm{IgG}_{4}$ response to birch pollen was down-regulated in nonatopic children but not in atopic children.

In contrast, the immune responses to inhalant allergens in atopic individuals are characterized by $\operatorname{IgE}$ antibody formation and T-cell responses, with the release of Th2-type cytokines. The results of a very recent study (Prescott et al. 1998) show that the atopic phenotype is associated with a prolonged period of Th2-type immune responses to allergens early in life. The findings confirm, at the T-cell level, previous observations that temporary low-level $\mathrm{IgE}$ responses to food and inhalant allergens are common during the first years of life, but are then down-regulated in nonatopic individuals and continue to increase in children who develop allergic manifestations. The difference between individuals with and without a genetically-determined atopic propensity may thus be how readily the neonatal Th2-skewed immunity deviates towards a Th1-type response. Thus, it seems reasonable to look for postnatal environmental factors affecting the incidence of allergy, rather than to advocate allergen avoidance during pregnancy.

\section{Epidemiological aspects}

The increasing prevalence and severity of atopic diseases seen in industrialized countries (Burr et al. 1989; Åberg et al. 1995) are in marked contrast with the low prevalence of allergy among children in the formerly socialist countries of Europe with a life-style similar to that prevailing in Western Europe 30-40 years ago (von Mutius et al. 1994; Bråbäck et al. 1995). The increase may in part be caused by the major improvements in public health and personal hygiene practice, diet changes, reduction in average family size and improvements in general living standards, i.e. a changed overall exposure to microbial stimulation (Holt et al. 1997).

The environment has undergone major changes in recent years. Rather than devoting all interest towards risk factors enhancing sensitization, a better way would be to search for factors that enhance the induction of tolerance. The discussion on the impact of environmental changes on the incidence of asthma and other allergies has been limited mostly to the possible effects of a deteriorating air quality, poorly-ventilated houses and an increased exposure to certain allergens, notably house-dust mites. None of these factors can explain more than marginally the observed regional differences in the prevalence of allergic diseases. However, 'environment' is much more than emissions from traffic and exposure to mites. The concept of 'lifestyle' should therefore be expanded considerably (Table 1), since an altered lifestyle also includes dietary changes, the microbial environment, extensive travelling to new environments, stress and much more. Furthermore, the mother herself is a little discussed, but significant, 'environmental factor' in early infancy.

\section{The role of breast milk}

During early infancy there is a close immunological interaction between the mother and her offspring, through the breast milk, but relatively little is known about the exchange of immunological information. In addition to numerous components that help in the protection against infection, human milk contains components that enhance the maturation of the immune system of the newborn infant (Duchén, 1999). Observations include an early stimulation of IgA antibody synthesis in breast-fed infants (Allardyce, 1984) and transfer of cell-mediated immunity and cytokines (Wold \& Adlerberth, 1998). Thus, human milk would not only provide passive protection against infections, but also actively stimulate infant immunity. However, there are considerable individual variations in the composition of human milk. This finding may explain the controversy with regard to the possible allergy-preventive effects of breastfeeding. If individual variations in the milk modulate the development of immunity in the neonate, then maternal immunity may represent an environmental factor which would influence the risk for allergic manifestations in her child, possibly even several years later.

Similar to the anti-infective properties, an anti-allergic effect by secretory IgA antibodies in human milk has been proposed. Low levels of total $\operatorname{IgA}$ and $\operatorname{IgA}$ antibodies against cow's milk have been reported in milk from mothers of food-allergic infants (Machtinger \& Moss, 1986), while in a larger prospective study this finding was not confirmed (Fälth-Magnusson et al. 1988). A disturbed composition of

Table 1. Some examples of changes in 'lifestyle' and environment that may possibly have affected the incidence of sensitization to allergens after the Second World War in industrialized countries with a market economy

\begin{tabular}{ll}
\hline $\begin{array}{l}\text { Urbanization } \\
\text { Diet }\end{array}$ & $\begin{array}{r}\text { Major changes in habits, exposure to air pollution } \\
\text { Foods that are new to the area, a wider range of } \\
\text { foods, food additives, foods processed by industry, } \\
\text { altered intestinal microbial flora }\end{array}$ \\
Buildings & $\begin{array}{r}\text { New building materials, more efficient insulation, } \\
\text { altered methods for construction, construction all } \\
\text { year round, even in temperate climates, leading to }\end{array}$ \\
& increased humidity \\
Homes & Larger dwellings, many new compounds at home \\
& (chemicals), increased indoor humidity in temper- \\
& ate and cold climates \\
Lifestyle & Most time spent indoors, extensive travelling and \\
& exposure to new environments
\end{tabular}


polyunsaturated fatty acids has been reported in milk from mothers of atopic infants. For example, the levels of the essential fatty acid linoleic acid, as well as its metabolites, were lower in early mature milk from atopic mothers as compared with non-atopic mothers, and $n 6: n-3$ fatty acids was higher (Yu et al. 1998). Differences in the fatty acid composition of human milk seems to have a relevance for the breast-fed infants. Thus, lower levels of the $n-3$ fatty acids, eicosapentaenoic acid, docosapentaenoic acid and docosahexaenoic acid, have been observed in mothers of infants who developed allergic disease during the first year of life as compared with mothers of babies who did not develop any allergic manifestations (Duchén et al. 1998). These differences were independent of maternal allergy. Similarly, low levels of certain polyamines have been observed in milk from atopic mothers (Duchén, 1999). As these compounds are required for optimal DNA synthesis, variations in the levels could affect immune responses to foreign antigens in breast-fed infants.

\section{The role of microbes}

It is well established that infections and other microbial pressure may drive the immune system towards a Th1-like response. Furthermore, it has been suggested that recurrent infections may enhance skewing towards Th1-type responses by exerting a pressure on the immune system (Holt et al. 1997). It would seem unlikely, however, that a stimulus that is potentially harmful to the host should be necessary for the postnatal maturation of a balanced immune system. Furthermore, the suggestions were based on epidemiological studies, and the evidence for a relationship between respiratory tract infections and protection against allergy is circumstantial. The observed relationships may, therefore, be spurious. For example, a suggested relationship between the exposure to tuberculosis and a low prevalence of allergy was based on the observation that allergic individuals less often had strongly positive tuberculin reactions, as compared with non-allergic persons (Shirakawa et al. 1997). However, this observation is more likely to be due to the fact that atopic individuals have depressed cell-mediated immune responses, including delayed-type hypersensitivity (Strannegård et al. 1998). When smaller tuberculin reactions were included in the comparisons, the atopic and non-atopic individuals had a similar prevalence of tuberculin positivity. Weak or absent tuberculin reactivity would, therefore, be merely an indication of a Th2-skewed immunity, and should not be regarded as an indication of less exposure to mycobacteria.

The gut flora is quantitatively the most important source of microbial stimulation, and may provide a primary signal for driving the postnatal maturation of the immune system, thus inducing Th1-like immunity (Rook \& Stanford, 1998). We have recently reported considerable differences in the composition of the gut flora of healthy 1-year-old infants in Estonia (which has a low prevalence of allergy; Bråbäck et al. 1995), and Sweden (Sepp et al. 1997). For example, lactobacilli and eubacteria were more common and the counts were higher in Estonian infants than in Swedish infants, whereas the reverse was true for Clostridium difficile. The postnatal colonization seems to be more intense in Estonian babies than in Swedish babies, as the counts of aerobes, particularly staphylococci, enterococci and enterobacteria, are much higher in the former (Sepp et al. 1999). At 1 month, the bacterial counts in the Swedish babies have reached the same level as in the Estonian infants. At that age, however, 80 $\%$ of Estonian infants, as compared with only $30 \%$ of Swedish babies, are colonized with lactobacilli. Very recently, differences in the composition of the intestinal microflora were demonstrated between allergic and nonallergic 2-year-old children, both in Estonia and Sweden (Björkstén et al. 1999). Thus, lactobacilli and bifidobacteria were more prevalent in the non-allergic children, while the counts of coliform bacteria were higher in the atopic children.

An alternative approach in the study of the microbial flora is to investigate the functional status of the flora, i.e. 'what have the microbes done?' A microflora-associated characteristic has been defined as any anatomical, physiological, immunological or biochemical function in a macroorganism which has been acted on by the microflora (Midtvedt, 1985). Examples of microflora-associated characteristics include the formation of short-chain fatty acids by anaerobic microbes in the human colon, microbial transformation of cholesterol to coprostanol, and the microbial inactivation of intestinal trypsin (EC 3.4.21.4) which can be measured by the remaining faecal tryptic activity. This approach to the study of the gut flora has several advantages over more traditional analyses based on isolation and enumeration of the micro-organisms, as the latter are expensive, time-consuming and associated with problems in obtaining samples from parts of the gastrointestinal tract that are normally inaccessible.

Employing this approach, an analysis of short-chain fatty acids was performed in stool samples from twenty-five allergic and forty-seven non-allergic Swedish infants (MF Böttcher, A Sandin, E Norin, T Midtvedt \& B Björkstén, unpublished results). Isocaproic acid was detected almost exclusively in allergic infants. This compound is associated with the presence of Clostridium difficile. In contrast, the levels of several fatty acids associated with a Lactobacillus flora were higher in the non-atopic infants.

The studies indicate an imbalance in the gut flora of allergic infants. As the microbial flora is driving the maturation of the immune system, changes in its composition, as a consequence of an altered lifestyle and diet in industrialized societies, may play a role in the higher prevalence of allergy. All these findings could suggest that differences in the indigenous intestinal flora might affect the development and priming of the immune system in early childhood, and that the observed differences between Estonian and Swedish infants, and between allergic and non-allergic children are not secondary phenomena.

It has been suggested that certain strains of lactobacilli, e.g. Lactobacillus casei, can inhibit allergen-induced $\operatorname{IgE}$ production by murine splenocytes, possibly through induction of interleukin 12 secretion by macrophages (Shida et al. 1998). Thus, the intestinal flora may play a crucial role in generating Th2 cell populations fully susceptible to oral tolerance induction (Sudo et al. 1997). Furthermore, the severity of atopic eczema improved in infants treated with extensively-hydrolysed whey formula fortified with Lactobaculus GG (Majamaa \& Isolauri, 1997).

Interestingly, products from lactobacilli-degraded casein proteins from cow's milk have been shown to stimulate or 
inhibit lymphocyte proliferation and interleukin 4 production, and enhance interferon $\gamma$ production (Shida et al. 1998). As lactobacilli are more common in Estonian children than in Swedish children, it is tempting to speculate that the gut flora of the Estonian children may be able to down-regulate Th2-like responses to cow's milk. In Estonian children atopic symptoms were associated with a trend towards high levels of $\mathrm{IgG}_{4}$ antibodies to birch pollen at 12 months, whereas there was a reverse tendency at 24 months (M Jenmalm and B Björkstén, unpublished results). This finding could be explained by a transiently increased Th2like response early in life in atopic children as compared with non-atopic children, which later disappears.

\section{Conclusions}

The major lifestyle and environmental factors triggering sensitization probably exert much of their influence during the first few years of life. The conclusion from a broad compilation of what is known regarding triggers of allergy is that we cannot identify which environmental factors are the major reasons for the large increase in the prevalence in recent years. The future search for significant environmental factors should be directed towards other areas that have not yet been explored. The intestinal microflora is one of these factors that deserve a closer analysis. Future research should be truly interdisciplinary, and 'lifestyle' should be given a broad interpretation.

\section{References}

Åberg N, Hesselmar B, Åberg B \& Eriksson B (1995) Increase of asthma, allergic rhinitis and eczema in Swedish schoolchildren between 1979 and 1991. Clinical and Experimental Allergy 25, 815-819.

Allardyce R (1984) Breast milk cell supernatants from atopic donors stimulate cord blood $\operatorname{IgA}$ secretion in vitro. Clinical Allergy 14, 259-267.

Björkstén B (1997) The environment and sensitisation to allergens in early childhood. Pediatric Allergy and Immunology $\mathbf{8}$, Suppl. $10,32-39$.

Björkstén B, Naaber P, Sepp E \& Mikelsaar M (1999) The intestinal microflora in allergic Estonian and Swedish 2-year old children. Clinical and Experimental Allergy (In the Press).

Björkstén F \& Suoniemi I (1981) Time and intensity of first pollen contacts and risk of subsequent pollen allergies. Acta Medica Scandinavica 209, 299-303.

Bråbäck L, Breborowicz A, Julge K, Knutsson A, Riikjäru M-A, Vasar M \& Björkstén B (1995) Risk factors for respiratory symptoms and atopic sensitization in the Baltic area. Archives of Disease in Childhood 72, 487-493.

Burr M, Butland B, King S \& Vaughan-Williams E (1989) Changes in asthma prevalence: two surveys fifteen years apart. Archives of Disease in Childhood 64, 1452-1456.

Croner S \& Kjellman N-I (1986) Predictors of atopic disease: cord blood IgE and month of birth. Allergy 41, 68-70.

Duchén K (1999) Human milk factors and atopy in early childhood. Medical Dissertation no. 580, University of Linköping, Sweden.

Duchén K, Yu G \& Björkstén B (1998) Atopic sensitisation during the first year of life in relation to long chain polyunsaturated fatty acid levels in human milk. Pediatric Research 44, 478-484.

Fälth-Magnusson K, Kjellman N-I \& Magnusson K (1988) Antibodies IgG, IgA and IgM to food antigens during the first 18 months of life in relation to feeding and atopic disease. Journal of Allergy and Clinical Immunology 81, 868-875.

Holt P, Sly P \& Björkstén B (1997) Atopic versus infectious disease in childhood: a question of balance? Pediatric Allergy and Immunology $8,1-5$.

Jenmalm M \& Björkstén B (1999) Exposure to cow's milk during the first three months of life results in failure to downregulate IgG subclass antibodies to B-lactoglobulin up to eight years. Journal of Allergy and Clinical Immunology (In the Press).

Julge K (1998) Humoral immune responses to allergens in early childhood. Medical Dissertation no. 558, University of Linköping, Sweden.

Machtinger S \& Moss R (1986) Cow's milk allergy in breastfed infants: the role of allergen and maternal secretory IgA antibody. Journal of Allergy and Clinical Immunology 77, 341-347.

Majamaa H \& Isolauri E (1997) Probiotics: a novel approach in the management of food allergy. Journal of Allergy and Clinical Immunology 99, 179-185.

Midtvedt T (1985) Microflora-associated characteristics (MACs) and germfree animal characteristics (GACs) in man and animals. Microecology and Therapy 15, 295-302.

Prescott S, Macaubas C, Holt B, Smallacombe T, Loh R, Sly PD \& Holt PG (1998) Transplacental priming of the human immune system to environmental allergens: universal skewing of initial T-cell responses toward the Th2 cytokine profile. Journal of Immunology 160, 4730-4737.

Rook G \& Stanford J (1998) Give us this day our daily germs. Immunology Today 19, 113-116.

Sepp E, Julge K, Vasar M, Naaber P, Björkstén B \& Mikelsaar M (1997) Intestinal microflora of Estonian and Swedish infants. Acta Paediatrica 86, 956-961.

Sepp E, Naaber P, Voor T, Mikelsaar M \& Björkstén B (1999) Development of intestinal micro-flora during the first month of life in Estonian and Swedish infants. Journal of Microbiology, Ecology and Health (In the Press).

Shida K, Makino K, Morishita A, Takamizawa K, Hachimura S \& Ametani A (1998) Lactobacillus casei inhibits antigen-induced $\operatorname{IgE}$ secretion through regulation of cytokine production in murine splenocyte cultures. International Archives of Allergy and Immunology 115, 278-287.

Shirakawa T, Enomoto T, Shin-ichiro S \& Hopkin J (1997) The inverse association between tuberculin responses and atopic disorder. Science 275, 77-79.

Strannegård I-L, Larsson L-O, Wennergren G \& Strannegård Ö (1998) Prevalence of allergy in children in relation to prior BCG vaccination and infection with atypical mycobacteria. Allergy $\mathbf{5 3}$, 249-254.

Sudo N, Sawamura S, Tanaka K, Aiba Y, Kubo C \& Koga Y (1997) The requirement of intestinal bacterial flora for the developement of an IgE production system fully susceptible to oral tolerance induction. Journal of Immunology 159, 1739-1745.

von Mutius E, Martinez FD, Fritzsch C, Nicolai T, Roell G \& Thiemann HH (1994) Prevalence of asthma and atopy in two areas of West and East Germany. American Journal of Respiratory and Critical Care Medicine 149, 358-364.

Wegmann T, Lin H, Guilbert L \& Mosmann T (1993) Bidirectional cytokine interactions in the maternal-fetal relationship: is successful pregnancy a Th2 phenomenon? Immunology Today 14, 353-356.

Wold A \& Adlerberth I (1998) Does breastfeeding affect the infant's immune responsiveness? Acta Paediatrica 87, 19-22.

Yu G, Duchén K \& Björkstén B (1998) Fatty acid composition in colostrum and mature milk from atopic and non-atopic mothers during the first 6 months of lactation. Acta Paediatrica 87, 729-736. 